\title{
Application of bisphosphomide-palladium(II) pincer complex in Suzuki-Miyaura cross-coupling reaction under microwave irradiation
}

\author{
MARUTHAI KUMARAVEL, PAWAN KUMAR and MARAVANJI S BALAKRISHNA* \\ Phosphorus Laboratory, Department of Chemistry, Indian Institute of Technology Bombay, Powai, \\ Mumbai 400 076, India \\ e-mail: krishna@chem.iitb.ac.in
}

MS received 13 September 2013; revised 20 November 2013; accepted 20 November 2013

\begin{abstract}
The bisphosphomide-based pincer complex $\left[\mathrm{PdBr}\left\{2,6-\left\{\mathrm{Ph}_{2} \mathrm{PC}(\mathrm{O})\right\}_{2}\left(\mathrm{C}_{6} \mathrm{H}_{3}\right)\right\}\right](2)$ has shown very high catalytic activity in Suzuki-Miyaura cross coupling reaction under microwave irradiation for a variety of aryl bromides and aryl boronic acids. The complex showed the same efficiency for gram scale reactions.
\end{abstract}

Keywords. Pincer complex; bisphosphomide; Suzuki-Miyaura coupling; microwave irradiation; palladium(II).

\section{Introduction}

The recent focus of interest on pincer complexes is due to their extended utility in the field of homogeneous catalysis. ${ }^{1}$ Pincer complexes offer interesting possibilities both in terms of mechanistic understanding and catalytic performance. ${ }^{1 b}$ The high catalytic efficiency is attributed to the stable and compact geometry of the pincer complexes with ideal steric and electronic properties. Among the cross coupling reactions, SuzukiMiyaura reaction is more popular because of its functional group tolerance for a wide variety of substrates. ${ }^{2}$ Microwave-assisted organic synthesis is becoming very useful and popular method due to its rapid reaction time, reproducibility and easy scale up methodologies in research laboratories and also in industrial processes. $^{3}$

We recently reported the synthesis of first examples of bis(phosphine), 1,3-phenylenebis((diphenylphosphino)methanone), $\left\{2,6-\left\{\mathrm{Ph}_{2} \mathrm{PC}(\mathrm{O})\right\}_{2}\left(\mathrm{C}_{6} \mathrm{H}_{3}\right)\right\}$ (1) (here after referred as bis(phosphomide)) and its transition metal chemistry and catalytic utility in hydroformylation reactions. ${ }^{4 a}$ Bisphosphomide $\mathbf{1}$ is a moderate $\sigma$-donor but a very good $\pi$-acceptor. This feature is ideal for facilitating the reductive elimination step in the catalytic cycle of cross-coupling reactions. ${ }^{4}$ As a part of our interest in designing the novel catalysts for organic transformations, ${ }^{5}$ we report here the catalytic utility of pincer complex, $\left[\mathrm{PdBr}\left\{2,6-\left\{\mathrm{Ph}_{2} \mathrm{PC}(\mathrm{O})\right\}_{2}\left(\mathrm{C}_{6} \mathrm{H}_{3}\right)\right\}\right]$ (2) in microwave-assisted Suzuki-Miyaura cross-coupling

\footnotetext{
*For correspondence
}

reactions of aryl and heteroaryl halides (figure 1). To the best of our knowledge, this is the first report of a pincer complex employed in microwave-assisted catalytic reactions.

\section{Experimental}

All bromo compounds and boronic acid derivatives were purchased from Aldrich. Anhydrous $\mathrm{K}_{2} \mathrm{CO}_{3}$ were purchased from SDFINE Chemicals, and used as received without further purification. Methanol, distilled over magnesium cake, was used for all catalytic reactions. All other reagents were used as received. The complex $\mathbf{2}$ was prepared according to literature procedure. ${ }^{\text {a }}$ Microwave experiments were carried out using a CEM Discover Labmate-microwave apparatus. GC analyses were conducted using an Agilent Gas Chromatograph 6890 Series, Hewlett Packard equipped with an HP5-MS capillary column $(30 \mathrm{~m} \times 0.25 \mathrm{~mm} \times$ $0.25 \mu \mathrm{m})$ and an FID detector. All GCMS analyses were done by Agilent 7890A GC system connected with 5975C inert XL EI/CI MSD (with triple axis detector).

\subsection{General procedure for the coupling reaction}

A mixture of 4-bromotoluene $(0.050 \mathrm{~g}, 0.291 \mathrm{mmol}, 1$ eq) and phenylboronic acid (0.043 g, $0.35 \mathrm{mmol}, 1.2$ eq), $0.2 \mathrm{ml}$ of stock solution prepared from dissolving palladium complex 2 (0.002 g, $0.1 \mathrm{~mol} \%, 0.001 \mathrm{eq})$ in $2 \mathrm{~mL} \mathrm{CH}_{3} \mathrm{CN}, \mathrm{K}_{2} \mathrm{CO}_{3}(0.060 \mathrm{~g}, 0.436 \mathrm{mmol}, 1.5 \mathrm{eq})$ and methanol $(3 \mathrm{~mL})$ was heated under microwave 
<smiles>O=C(c1ccccc1)c1cccc(C(=O)c2ccccc2)c1</smiles>

1<smiles>O=C1c2cccc3c2[Te](Br)(Br)[P+]13P</smiles>

2
Figure 1. Structure of bisphosphomide based PCP palladium pincer complex 2 .

condition at $85{ }^{\circ} \mathrm{C}$ and 60 watt for 8 mins to give 4-methyl-1,1'-biphenyl. The substrate scope with different aryl halides and different boronic acids were tested using the same procedure as above. The typical molar ratio of the reaction components in all cases was 1/1.2/1.5/0.001/3 $\mathrm{mL}$ (aryl halide, aryl boronic acid, $\mathrm{K}_{2} \mathrm{CO}_{3}$, catalyst and methanol). The conversion with respect to aryl halide was determined by GC using dodecane as internal standard and products were confirmed by GC-MS.

\section{Results and discussion}

The pincer complex $\mathbf{2}$ was prepared by using the recently reported literature method. ${ }^{4 a}$ In order to obtain optimized conditions, initial catalytic investigations were carried out using 4-bromotoluene and phenylboronic acid as a model reaction. The effect of solvents and the reaction time was optimized using $0.1 \mathrm{~mol} \%$ of palladium catalyst and $\mathrm{K}_{2} \mathrm{CO}_{3}$ as a base. As summarized in table 1 , methanol was chosen as solvent. The complex 2 gave complete conversion (100\%) when the reaction time was extended to 8 min under microwave irradiation. Similar results were obtained when the reaction was performed under refluxing temperature (entry $8,2 \mathrm{~h}$ ) or at room temperature (entry $9,12 \mathrm{~h}$ ). Since $100 \%$ yields were obtained in $8 \mathrm{~min}$, microwave condition was employed. $\mathrm{K}_{2} \mathrm{CO}_{3}$ was used as a base due to its low cost and good efficiency.

To expand the scope of catalytic investigation to several substrates, complex $\mathbf{2}$ was tested with highly challenging ortho-substituted aryl bromides and also with substrates containing electron donating (deactivating) substituents using phenylboronic acid under optimized conditions. The results are summarized in table 2 . A very high TOF of $\approx 7000$ was obtained for methyl and methoxy substituted aryl bromides. Moderate to good conversions were observed for both $\mathrm{NMe}_{2}$ and methoxy substituents at the para position. 2,4Dimethoxy-bromobenzene with two methoxy groups showed a TOF of 4875 (entry 6).

Aryl bromides containing electron withdrawing (activating) groups at ortho position also showed almost quantitative conversions (entries 12-14). The

Table 1. Optimization of reaction conditions on Suzuki-Miyaura reaction of 4-bromotoluene with phenylboronic acid ${ }^{\mathrm{a}}$.

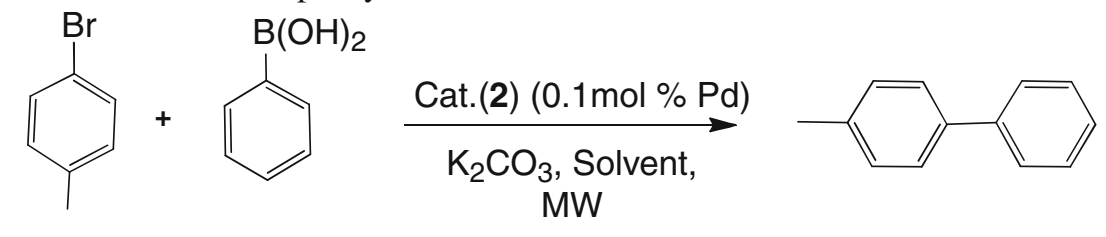

\begin{tabular}{lcccc}
\hline Entry & Solvent & $\begin{array}{c}\text { Temperature } \\
(\text { deg C })\end{array}$ & $\begin{array}{c}\text { Time } \\
(\mathrm{min})\end{array}$ & $\begin{array}{c}\text { Conversion }^{\mathrm{b}} \\
(\%)\end{array}$ \\
\hline 1 & Methanol & 85 & 5 & 88 \\
2 & Toluene & 110 & 5 & 56 \\
3 & THF & 66 & 5 & 3 \\
4 & $\mathrm{CH}_{3} \mathrm{CN}$ & 85 & 5 & 24 \\
5 & Dioxane & 100 & 5 & 61 \\
6 & DMF & 130 & 5 & 55 \\
7 & Methanol & $\mathbf{8 5}$ & $\mathbf{8}$ & $\mathbf{1 0 0}$ \\
$8^{\mathrm{c}}$ & Methanol & 70 & $2 \mathrm{~h}$ & 98 \\
9 & Methanol & $\mathrm{RT}$ & $12 \mathrm{~h}$ & 94 \\
\hline
\end{tabular}

${ }^{\mathrm{a}}$ Reaction was performed in a sealed tube containing aryl bromides $(0.291 \mathrm{mmol})$, aryl boronic acid $(0.349 \mathrm{mmol} ; 1.2 \mathrm{eq})$, base $(0.436 \mathrm{mmol} ; 1.5$ eq.), catalyst $(0.1 \mathrm{~mol} \%)$ and solvent $(3 \mathrm{~mL})$. An initial microwave power of $60 \mathrm{~W}$ was applied to reach $85^{\circ} \mathrm{C}$ temperature.

${ }^{\mathrm{b}}$ Conversions were determined by GC using aryl halides as internal standards.

${ }^{\mathrm{c} C}$ Conventional heating was carried out using an oil bath 
Table 2. Suzuki-Miyaura reaction ${ }^{\mathrm{a}}$ of phenylboronic acid with various aryl halides.
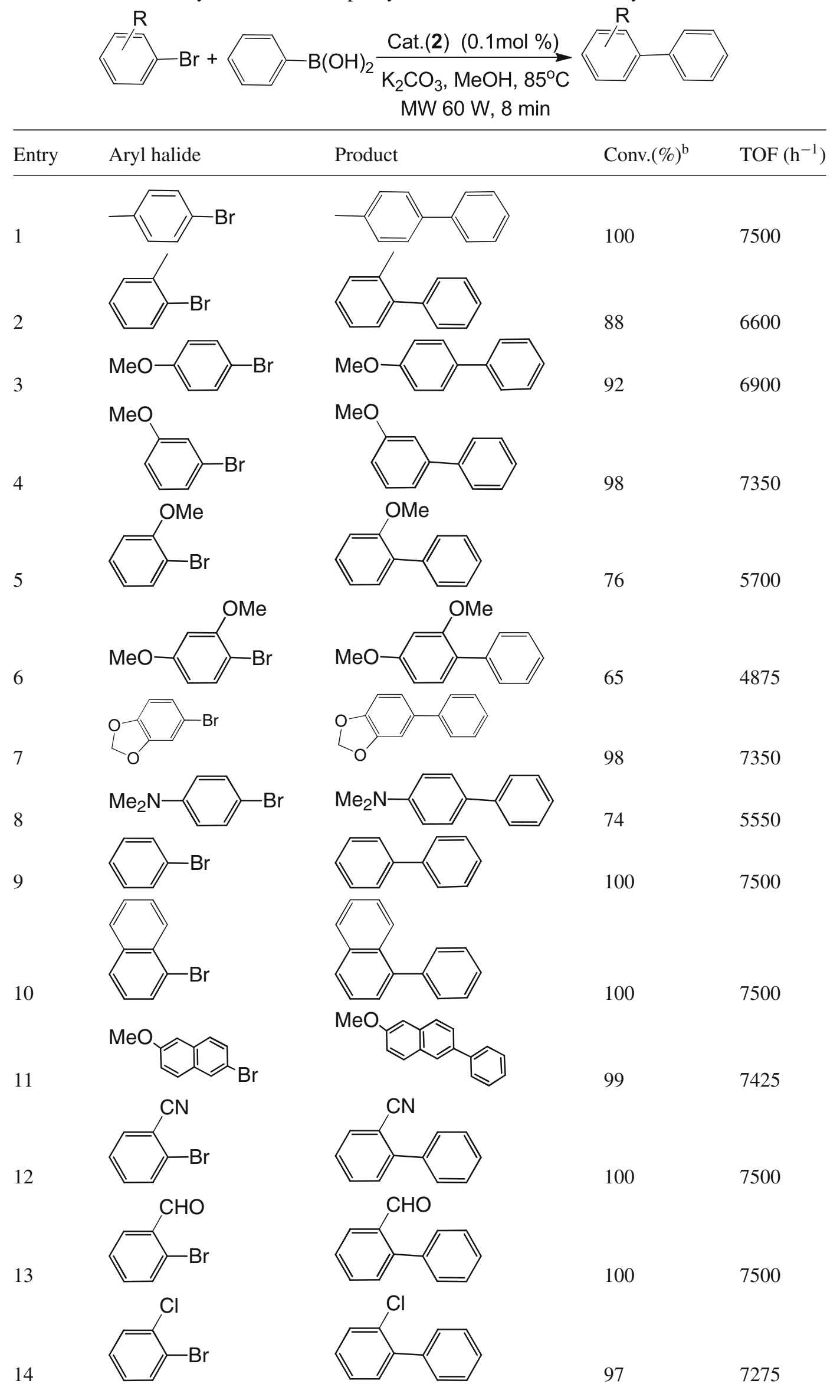
Table 2. (continued)

Entry

${ }^{\mathrm{a}, \mathrm{b}}$ Reaction conditions are similar to those described in table 1

Table 3. Suzuki-Miyaura reaction ${ }^{\mathrm{a}}$ of 4-bromotoluene with various arylboronic acid derivatives.

Entry


Table 3. (continued)

Entry Aryl boronic acid

${ }^{\mathrm{a}, \mathrm{b}}$ Reaction conditions are similar to those described in table 1.

conversions of heteroaromatic bromides such as bromopyridines and bromothiophene (entries 16-19) were excellent despite their ability to coordinate to the metal centre and hinder the catalytic process. Further, the homo-coupling of phenylboronic acids a very common and usual side reaction is not observed in all these cases.

In order to establish the efficiency and versatility of the precatalyst $\mathbf{2}$, different aryl boronic acids have been tested and the results are summarized in table 3 . It is well-known that the boronic acids with electron donating substituents are more nucleophilic than electron withdrawing ones and perform better in the coupling reaction as compared to the later ones. As expected, the complex $\mathbf{2}$ catalysed methyl and methoxy substituted boronic acids efficiently, whereas the relative catalytic performance with respect to the electron withdrawing substituents was moderate (entries 25-30) with a TOF of 6900 for 4-fluorophenylboronic acid.

The actual catalytic performance of a complex is decided by its effectiveness in scale-up reactions and its consistent reproducibility. Catalysts which perform well in large scale reactions are very important for pharmaceutical and chemical industries. The reaction between $1 \mathrm{~g}$ of $p$-bromotoluene and 1.2 equivalent of phenylboronic acid in the presence of complex $\mathbf{2}$ yielded the coupled product in $78 \%$ within 30 min. ${ }^{6}$

\section{Conclusions}

The initial investigations of bisphosphomide-palladium pincer complex 2 in Suzuki-Miyaura cross-coupling reaction have been very successful. A very high TOF was achieved even for sterically hindered and deactivated aryl halides and good yields were obtained in scale-up reactions. Further utility of this complex towards various other organic transformations are under investigation in our laboratory.

\section{Acknowledgements}

We thank the Department of Science and Technology (DST), New Delhi, for financial support. MK thanks the Council of Scientific Industrial Research (CSIR), New Delhi, for Senior Research Fellowship (SRF).

\section{References}

1. (a) van der Boom M E and Milstein D 2003 Chem. Rev. 103 1759; (b) Morales-Morales D and Jensen C M (eds) 2007 The chemistry of pincer compounds (Amsterdam: Elsevier); (c) Morales-Morales D 2008 Mini-Rev. Org. Chem. 5 141; (d) Selander N and Szabó K J 2011 Chem. Rev. 111 2048; (e) Beletskaya I P and Cheprakov A V 2004 J. Organomet. Chem. 6894055

2. (a) Miyaura N and Suzuki A 1995 Chem. Rev. 95 2457; (b) Bedford R B, Draper S M, Scully P N and Welch S L 2000 New J. Chem. 24 745; (c) Garagorri D B, Bocokić V, Mereiter K and Kirchner K 2006 Organometallics 25 3817

3. (a) Sajith A M and Muralidharan A 2012 Tetrahedron Lett. 53 1036; (b) Hajipour A R, Karami K and Pirisedigh A 2011 Inorg. Chim. Acta 370 531; (c) Susanto W, Chu C -Y, Ang W J, Chou T -C, Lo L -C and Lam Y 2012 Green Chem. 1477

4. (a) Kumar P, Siddiqui M M, Reddi Y, Mague J T, Sunoj R B and Balakrishna M S 2013 Dalton Trans. 42 11385; (b) Gowrisankar S, Federsel C, Neumann H, Ziebart C, Jackstell R, Spannenberg A and Beller M 2013 Chem. Sus. Chem. 6, 85; (c) Whittemore S M, Yoder R J and Stambuli J P 2012 Organometallics 316124

5. (a) Balakrishna M S, Rao S and Choubey B 2012 J. Chem. Sci. 1241191 and references there in; (b) Balakrishna M S, Suresh D and Mague J T 2011 Inorg. Chim. Acta 372 259; (c) Ganeshamoorthy C, Mague J T and Balakrishna M S 2008 Eur. J. Inorg. Chem. 596; (d) Ganeshamoorthy C, Mague J T, Balakrishna M S and Tuononen $\mathrm{H}$ M 2008 Inorg. Chem. 47 7035; (e) Venkateshwaran R, 
Balakrishna M S and Mobin S M 2007 Eur. J. Inorg. Chem. 1930; (f) Punji B, Mague J T and Balakrishna M S 2007 Inorg. Chem. 47 10268; (g) Punji B, Mague J T and Balakrishna M S 2007 Inorg. Chem. 47 11316; (h) Rao S, Mague J T and Balakrishna M S 2013 Dalton Trans. 42 11695

6. For large scale reaction: A mixture of 4-bromotoluene (1.044 g, $6.1 \mathrm{mmol}, 1 \mathrm{eq})$, phenylboronic acid (0.893 g, $7.3 \mathrm{mmol}, 1.2 \mathrm{eq}), \mathrm{K}_{2} \mathrm{CO}_{3}(1.266 \mathrm{~g}, 9.16 \mathrm{mmol}, 1.5 \mathrm{eq})$, complex $2(0.0042 \mathrm{~g}, 0.1 \mathrm{~mol} \%, 0.001 \mathrm{eq})$ and methanol
(10 $\mathrm{mL})$ taken in a $25 \mathrm{~mL}$ reactor vessel was heated under microwave condition at $85^{\circ} \mathrm{C}$ and 60 watt for 30 min. After the completion of the reaction, the product (4-methylbiphenyl) obtained was separated and purified by column chromatography using 5:1 mixture of ethyl acetate and petroleum ether (b.p. range $60-85^{\circ} \mathrm{C}$ ). Yield $78 \%$ (0.800 g, $4.78 \mathrm{mmol}){ }^{1} \mathrm{H}$ NMR (400 MHz, $\left.\mathrm{CDCl}_{3}\right) \delta$ $7.64(\mathrm{~d}, J=7.1 \mathrm{~Hz}, 2 \mathrm{H}), 7.55(\mathrm{~d}, J=8.1 \mathrm{~Hz}, 2 \mathrm{H}), 7.48$ $(\mathrm{t}, J=7.5 \mathrm{~Hz}, 2 \mathrm{H}), 7.37(\mathrm{t}, J=7.3 \mathrm{~Hz}, 1 \mathrm{H}), 7.30(\mathrm{~d}$, $J=7.9 \mathrm{~Hz}, 2 \mathrm{H}), 2.45$ (s, 3H). Mp. $44-47^{\circ} \mathrm{C}$ 